\title{
Les papiers de la guerre, la guerre des papiers ${ }^{1}$
}

\author{
Par Stéphane Michonneau \\ Université de Poitiers
}

Le 29 décembre 2004, le chef du Parti populaire actuellement dans l'opposition, Mariano Rajoy, déclarait que "pour sortir un seul document [des archives historiques] de Salamanque, il faudra [lui] passer sur le corps" ${ }^{2}$. Quelques jours plus tard, le maire de la ville castillane, Julián Lanzarote, fit élever autour du bâtiment des archives une palissade officiellement destinée à des travaux de réfection du pavement des rues du quartier. Le président des Cortes de la région autonome de Castille-Léon, Juan Vicente Herrera, déclarait à son tour, la gorge serrée, que l'atteinte faite aux Archives de la guerre civile équivalait au "démantèlement et au démembrement de l'Espagne". Rien de moins.

L'affaire des "papiers de Salamanque" qu'un arbitrage de l'État semblait avoir clos après 25 ans de conflits ne semble pas devoir se terminer. Par l'une de ces polémiques dont la vie politique espagnole a le secret, l'affaire reprend de plus belle, faisant les choux gras de la presse et des multiples associations formées pour l'occasion. Rien que dans El País, on ne compte pas moins d'une soixantaine d'articles enflammés depuis septembre 2004. Des manifestations rassemblant des milliers de citoyens se mobilisent régulièrement en divers lieux d'Espagne. L'émotion est à son comble. Comment 507 cartons d'archives pourraient-ils mettre en péril l'unité de l'Espagne, si l'on en croit l'opposition, ou tout du moins fragiliser durablement le gouvernement socialiste de José Luis Zapatero?

Si le scandale des "papiers de Salamanque" remonte aux premiers jours de la transition démocratique, en 1977, c'est parce que les documents déposés dans le couvent Saint- Ambroise à Salamanque proviennent du pillage systématique effectué en 1939 par les troupes franquistes dans la zone républicaine, dans le dessein de réprimer férocement l'ennemi "rouge". Ce centre de documentation policier et judiciaire, qui

\footnotetext{
${ }^{1}$ La "guerre des papiers" est une expression d'Eduardo Haro Tecglen dans El País, 31/12/2004.
} 
rappelle celui de la Stasi en RDA, inscrit à la hâte au registre des archives nationales sous le titre abusif de "Archives de la guerre civile" en 1977, a fait l'objet de nombreuses demandes de restitutions, notamment de la part du gouvernement autonome de Catalogne, la Generalitat, après son rétablissement en 1980. Si le principe du retour des archives catalanes fut acquis dès cette époque, le transfert de la documentation ne se réalisa jamais, laissant la situation se détériorer au point de constituer une pomme de discorde entre les Espagnols d'aujourd'hui. "Parler de pillage des documents déposés à Salamanque, c'est parler de la guerre civile" avoue le porte-parole du parti catalaniste conservateur Convergència $i U_{n i o ́}{ }^{3}$. C'est aussi parler de la période noire de la répression franquiste dont les Espagnols découvrent aujourd'hui l'ampleur avec effarement. L'Espagne, une fois encore, doit faire face à ses fantômes, soixante ans après les événements.

\section{Archives ou spoliations?}

Les conditions très particulières de constitution des Archives de la guerre civile ne font pas de ce centre un dépôt comme les autres ${ }^{4}$. Dès le 13 décembre 1936, le décret franquiste qui interdisait les partis politiques ordonnait la confiscation de la documentation de ces formations. À partir d'avril 1937, divers organismes furent chargés de concentrer la documentation des zones récemment "libérées", sous l'égide du bureau de recherche et de propagande antimarxiste (OIPA) dépendant directement du chef d'État. En mai de la même année fut créée la délégation des affaires spéciales (DAE) dédiée uniquement à la contre-propagande antimaçonnique. Ces deux entités fusionnèrent en avril 1938, une fois le premier gouvernement formel de l'Espagne nationale nommé, dans la délégation pour la récupération de documents (DERD), dirigée par Marcelino de Ulibarri et dépendant du ministère de l'Intérieur. L'objectif était de réunir la documentation "susceptible de prouver les antécédents et les agissements des ennemis de l'État [...] et de fournir des faits utiles à tous les organismes

\footnotetext{
${ }^{2}$ El País, 29/12/2004.

${ }^{3}$ El Mundo, 10/02/2005, "PSOE y nacionalistas tumban un pacto de Estado a favor de la unidad del Archivo de Salamanca".

${ }^{4}$ Ces informations sont tirées de Angels Bernals, Miquel Casademont, Antoni Mayans, La documentació catalana a Salamanca : un estat de la qüestio, 1936-2003, Barcelona, Associació d'Arxivers de Catalunya, 2003. Cet ouvrage comprend une bibliographie très complète sur les fonds des Archives générales de la guerre civile espagnole, sur l'histoire de la spoliation et de la revendication catalane et sur le problème archivistique général de la restitution des biens spoliés. Voir aussi Josep Cruanyes, Els
} 
chargés de sa défense" ${ }^{5}$. Ces dispositions ne laissent aucun doute quant à l'usage des papiers : la répression. L'appareil légal de la répression s'élabora à la même époque sur la base de la loi de responsabilité politique ( 9 février 1939), de la loi pour la répression de la maçonnerie et du communisme (1er mars) et de loi dite de "la Cause Générale" (26 avril) qui punissait "les délits commis sur le territoire national pendant la domination rouge". Les organismes chargés d'appliquer ces nouvelles directives furent donc les principaux usagers des archives, en particulier le Tribunal spécial pour la répression de la maçonnerie et le communisme (TERMC) entre 1940 et 1963. Si la libre consultation des fonds ne fut jamais permise, la vocation du centre ne changea officiellement qu'en 1977, lorsqu'il dépendit du ministère de la Culture.

À mesure que l'armée franquiste avançait en territoire ennemi, les équipes de récupération de la DERD opéraient systématiquement : à Bilbao, à Madrid, à Barcelone, à Valence entre autres. Pour Barcelone, l'historien Josep Cruanyes a établi le mécanisme de l'opération de pillage : deux jours après l'occupation militaire de la capitale catalane, le 28 janvier 1939, six équipes commencèrent leur travail jusqu'au 7 juin suivant, en visitant de 1700 à 1800 dépôts répartis dans la ville. La documentation accumulée alla jusqu'à occuper une douzaine de locaux réquisitionnés, garages, magasins, dépôts divers, où s'installèrent les services de catalogage chargés de relever les noms qui figuraient dans la documentation examinée. L'analyse des registres de confiscation montre l'extrême variété des provenances : départements et services de la Generalitat de Catalunya qui existait depuis 1932, organisations politiques et syndicales variées, archives de militants et du personnel politique républicains, correspondance des membres du gouvernement mais aussi de personnalités du monde de la culture, archives d'entités culturelles soupçonnées d'incarner des valeurs contraires à celles du nouvel État (Ateneus, loges, bibliothèques et universités populaires, etc.). De même, la DERD rassembla les publications, les périodiques, les journaux de toutes sortes entreposés dans les librairies et les imprimeries afin de connaître leurs auteurs et leurs souscripteurs ( $L a$ Batalla, La Humanitat, La Publicitat, Treball, La Veu de Catalunya, etc.). Les photographies servirent à confondre les personnes pourchassées.

papers de Salamanca. L'espoliació del patrimoni documental de Catalunya (1938-1939), Barcelona, Ed. 62 (c'est le nom de l'éditeur : Oui, c'est son nom), 2003.

${ }^{5}$ La documentació catalana..., op. cit., p.9 : "unificar e intensificar (...) la recogida, custodia y clasificación de todos aquellos documentos aptos para obtener antecedentes sobre las actuaciones de los 
Toute cette documentation ne partit pas pour Salamanque, première capitale de l'État franquiste et siège central de la DERD. D'abord, d'autres organismes concurrents se chargèrent de spolier toutes sortes d'archives, notamment la Phalange et l'armée d'occupation. Surtout, une grande quantité de papiers furent détruits ou abandonnés, parfois même rendus à leurs propriétaires. Une partie des fonds d'archives dispersés dans la ville de Barcelone put être sauvée lors de circonstances hasardeuses : l'exil, la conservation clandestine, la réaffectation des archives à de nouvelles entités mises en place en 1939, etc. Rassemblés aujourd'hui aux Archives nationales de Catalogne, ces documents représentent les trois quarts des fonds de la Generalitat républicaine. Pour le reste, les papiers furent transférés en Castille entre juin 1939 et février 1940, soit 3500 sacs contenant 140 tonnes d'archives. Mais à Salamanque aujourd'hui, il ne reste que $10 \%$ des archives spoliées en 1939. Là, elles furent divisées en de multiples magasins et en deux sections : la section spéciale s'occupait de la maçonnerie et la section politico-sociale des autres entités et associations. Rangés par zone géographique dans la plus grande confusion, les dossiers relèvent davantage de l'accumulation que d'une logique d'archivage raisonné. L'unique entrée de ce pot-pourri documentaire est le fichier nominal dont se servait la mécanique répressive de l'État. Le fichier de la section politico-sociale recense trois millions de personnes, celui des maçons, 180000.

La suppression du TERMC en 1963 signifia l'entrée en décadence du service de la DERD, devenue entre temps, la DNSD (délégation nationale de services documentaires) en 1944. La mort de Franco, en novembre 1975, sonna le glas d'une institution dont la transition n'avait nul besoin. La documentation passa donc sous la direction du ministère de la Culture en octobre 1977, puis fut inscrite au registre des Archives historiques nationales en mai 1979 à titre autonome, sous le nom de "Section de la guerre civile" (SGC). La SGC fut dirigée à partir de 1980 par un comité directeur de 18 membres. Le centre salamantin eut désormais pour fonction la reconnaissance des droits et des services prêtés dans les organes de la République de la part des personnes qu'il avait contribué à réprimer...

enemigos del Estado [...] y suministrar datos útiles a todos los demás organismos encargados de su defensa". Décret du 27 avril 1938. 
À dire vrai, la SGC ne constitua jamais un centre d'archives digne de ce nom. Le volume considérable de documents emmagasinés; la complexité d'organisation d'un fond qui n'avait pas été prévu pour la consultation, la méconnaissance de la réalité de la part des responsables et l'absence de moyens expliquent la sorte d'abandon dans lequel se trouvaient les fonds salamantins jusqu'à une date récente. L'hétérogénéité de la documentation, caractéristique majeure du fond, fut aggravée par l'absence complète de politique d'archivage cohérente tout au long des années 1980. Aucun plan archivistique général ne fut déterminé et l'accès fut des plus restreints, de sorte que le manque de transparence devint l'atout majeur d'un centre d'archives dont de nombreuses demandes de restitution remettaient en cause l'existence. Comment restituer une documentation dont il n'existe aucun registre fiable?

Le nom de la SGC porte à confusion car ce dépôt est loin de concentrer l'essentiel de la documentation concernant les événements du conflit civil ${ }^{6}$. L'ensemble des archives militaires se trouve par exemple à Ségovie, celle de la diplomatie à Madrid, celle de l'administration à Alcalá de Henares. Il existe dans les archives du Gouvernement de nombreux documents qui enrichiraient la connaissance de la guerre civile. Pas un papier concernant la Seconde République n'a naturellement quitté les Archives nationales à Madrid. Pour étudier la guerre, il faut de plus compter sur de très nombreuses fondations privées (Archivo Fal Conde à Séville, fondations Pemán à Madrid, Pablo Iglesias, Largo Caballero...), sur les fonds importants des Archives nationales de Catalogne et sur ceux de l'Institut d'histoire sociale d'Amsterdam. S'agissant à Salamanque de documents spoliés dans l'ancienne zone républicaine, il n'existe pas non plus un seul document se référant à la Galice, à la Castille, à l'Alava ou bien à la Navarre qui, dès juillet 1936, se rangèrent du côté du soulèvement militaire. Enfin, une partie des fonds salamantins fut transportée à Madrid pour nourrir la Causa general instruite par le procureur général du Tribunal suprême à partir de 1940.

Incomplètes pour la guerre civile, les archives salamantines comprennent en outre une importante documentation se référant "aux années antérieures au conflit" : on y trouve des correspondances personnelles datées de 1875, des livres d'actes d'associations 
culturelles des années 1910... Par exemple, le dossier 1389 portant la mention "Fomento del trabajo nacional - 1925" rassemble les papiers du plus important syndicat patronal catalan au milieu de dossiers "rouges-séparatistes", simplement parce que pendant la guerre, le syndicat anarchiste CNT occupa le bâtiment du Fomento (Via Laiaetana, 64). En 1939, les "équipes de récupération" enlevèrent le tout sans prêter gare au détail. En partant de l'unique registre disponible datant de 1948, le travail de catalogage n'est toujours pas terminé aujourd'hui. Jordi Busquets n'a pas tout à fait tort de considérer que la SGC relève davantage du magasin de dépôt que du centre d'archives ${ }^{7}$.

Dès l'origine, l'absence de catalogage rendit le problème des restitutions particulièrement épineux. L'instauration d'un régime provisoire d'autonomie pour la Catalogne par le gouvernement Suarez, dès 1977, reposa sur le principe d'une continuité institutionnelle entre la Generalitat républicaine et la nouvelle Generalitat, justifié par le maintien d'organes institutionnels en exil entre 1939 et $1977^{8}$. Le retour du président catalan Taradellas à Barcelone fut négocié à la condition que la Catalogne accepte le cadre institutionnel monarchique. En fait, la Generalitat est en Espagne l'unique institution officielle qui tire une partie de sa légitimité d'une loi de la République... C'est au nom de cette continuité que le premier gouvernement statutaire de Catalogne, constitué en avril 1980 après la promulgation de la constitution de 1978, réclama le retour de "ses" papiers. Si le gouvernement central avait de bonnes dispositions, il fallait en bonne logique terminer le catalogage des fonds. En 1980 d'ailleurs, un projet de loi avait été soumis au Parlement dans ce sens, deux jours avant les premières élections du Parlement de Catalogne, et ce par un représentant du parti de droite d'alors, dirigé par Manuel Fraga, ancien ministre de la communication de Franco. La proposition admettait le tort commis.

L'objectif de la convention signée en 1982 entre les services culturels de la Generalitat et le ministère de la Culture désormais socialiste était la reconnaissance et le microfilmage des fonds de la SGC. Financés par le gouvernement catalan, les travaux s'étendirent de mars 1983 à décembre 1986, sur la base d'une réorganisation physique

\footnotetext{
${ }^{6}$ El País, 09/01/2005, Javier Tusell, "¿Dónde están los papeles de la guerra?".

${ }^{7}$ El País, 02/11/2002, Jordi Busquets, "Salamanca : almacén que no archivo".

${ }^{8}$ La revendication de la Generalitat fut motivée entre autres par la mobilisation des archivistes en Catalogne lors du congrès qu'ils tinrent à Lleida au printemps 1976.
} 
des archives selon leur provenance. Au total, 507 dossiers furent constitués puis très lentement microfilmés, jusqu'en octobre 1993. Indice évident d'une réticence à peine voilée des autorités salamantines. C'est ainsi que le registre de confiscation de 1948 ne fut porté à la connaissance des archivistes catalans qu'en 1995, soit dix ans après qu'ils avaient terminé leur tâche, pour invalider leur travail !

La revendication de la Generalitat portait sur la documentation confisquée aux personnes physiques et juridiques catalanes, et non pas sur la documentation provenant des organismes répressifs en propre (L'OIPA, la DERD puis la DNSD). La demande ne concernait pas seulement les archives de la Generalitat républicaine mais aussi celles des loges maçonniques, de la presse, des documents personnels, des correspondances privées, etc., spoliées en 1939. Elle reposait sur le principe de l'unité des fonds et non pas sur celui de l'unité des Archives. En effet, l'unité de fond considère que les papiers générés par une personne forment un seul et unique ensemble documentaire. Les producteurs des documents et leurs héritiers en sont donc les uniques et légitimes titulaires ${ }^{9}$. La propriété ne se détermine donc ni par le type d'information contenu (des papiers se référant au conflit en l'occurrence), ni par l'usage ultérieur que l'on en fit (la répression), et moins encore par le lieu de dépôt (Salamanque). Dans le cas de la documentation des administrations publiques (Generalitat ou municipalités), les droits du titulaire n'ont pas de prescription.

Tout en reconnaissant la légitimité de la plainte, les premiers gouvernements démocratiques alléguèrent des objections techniques qui ajournèrent le retour des "papiers de Salamanque". La première objection, on l'a vu, portait sur la difficulté d'identifier les documents : le problème, au moins pour ce qui relève des archives de la Generalitat, fut réglé en 1993. À partir de cette date, le gouvernement socialiste défendit le principe de l'unité de la SGC, contraire à ceux de l'archivistique internationale. Le 15 mars 1991, l'Assemblée générale de l'ONU a effectivement voté

\footnotetext{
${ }^{9}$ L'archivistique contemple (est-ce un terme consacré en archivistique. Non, je ne crois pas, c'est un hispanisme... Pourrait-on mettre "admet"?))quelques exceptions en vertu desquelles des documents peuvent s'intégrer aux fonds d'un autre producteur que l'original : en cas de transfert de compétence d'un organisme à un autre, en cas de nécessité pour continuer l'exercice de la compétence (actes de fondation, actes de propriété). Exceptionnellement, l'ancienne documentation peut s'attribuer à la nouvelle administration. Ces mesures d'exceptions sont compréhensibles dans le contexte d'un État de droit et ne peuvent en aucun cas légitimer une activité frauduleuse, une agression ou un attentat contre le patrimoine documentaire. Enl'occurrence, on peut considérer que la DERD est un fond propre (ceux produits par l'institution de répression) auquel s'ajoute des fonds volés. C'est donc un fond de fonds.
} 
une résolution du Conseil international des archives manifestant le droit légitime des propriétaires à exiger la restitution de la documentation objet de vol ou de soustraction dans le cadre de conflits. C'est la raison pour laquelle en mars 1995 le gouvernement accorda à la Generalitat la reconnaissance de son droit légitime : le processus de restitution était en marche.

Contre la décision de la ministre de la Culture, Carmen Alborch, 55000 Salamantins défilèrent contre le transfert des "papiers" ${ }^{10}$. Le gouvernement socialiste aux abois suite à de nombreux scandales tenta alors de freiner le processus engagé : c'est dans ce contexte que surgit opportunément le registre de 1948 ! Cette même année, en 1996, le Congrès des députés demanda à quatre historiens de la Junte supérieure des archives de fournir un rapport définitif sur la base de la sauvegarde de l'unité thématique du centre d'archives ${ }^{11}$. Les conclusions préconisaient la création à Salamanque d'un grand centre d'archives sur la guerre civile qui porterait le nom des Archives générales de la Guerre Civile Espagnole (AGGCE). Doté de moyens supérieurs, le nouveau centre bénéficierait de centres de recherches, d'une bibliothèque, d'un centre d'histoire orale et de moyens de reproduction et d'achat sans commune mesure avec ceux de l'ancienne SGC. L'Étatmajor, le ministère de la Défense, les Archives nationales de Catalogne offrirent alors soit leurs documents, soit leurs reproductions, pour faire de Salamanque un grand centre d'étude du conflit. En échange, la Junte envisageait un système de dépôts de documents, à l'instar de ce que pratique le musée du Prado dans les musées de province. Il s'agissait bien d'un dépôt, non pas d'une restitution. La nature du dépôt dépendait entièrement du comité directeur de l'AGGCE, une fois établie une commission technique formée de représentants du comité et de la Generalitat ${ }^{12}$.

La tempête politique se calmait donc. Mais en mars 1996, le Parti populaire gagnait les élections législatives, mettant fin à douze années de gouvernement de felipismo. C'est la nouvelle ministre de la Culture, Esperanza Aguirre, qui reçut les conclusions du rapport que nous mentionnions plus haut.

\footnotetext{
${ }^{10}$ À cette époque, le maire de Salamanque, Jesús Málaga, était socialiste.

${ }^{11}$ Il s'agit d'Antonio Elorza, de Josep Fontana, de Santos Juliá et de Javier Tusell.

${ }^{12}$ El País, 26/07/2002, Javier Tusell, "Más que una cuestion de papeles de archivo".
} 
Une longue attente de quatre années motiva le vote, en juin 2000, d'une demande de restitution des "papiers" de la part du Parlement de Catalogne. Tous les groupes politiques furent unanimes, à l'exception du Parti populaire (PP) catalan. Le gouvernement refusa d'envisager ce transfert même s'il n'écartait pas la présence "hypothétique" des documents en Catalogne ${ }^{13}$. Les rapporteurs de 1996 dénoncèrent également la passivité et le silence des autorités de tutelle qui optaient évidemment pour l'enlisement. Antonio Elorza dénonçait les règles de nomination du comité directeur des archives salamantines, principalement composé d'élus locaux naturellement hostiles à toute restitution ${ }^{14}$. Le gouvernement ne pouvait donc que réunir la commission technique promise il y a quatre ans. Pour le PSOE désormais dans l'opposition, la restitution des archives devenait souhaitable.

La commission technique fut nommée en novembre $2000^{15}$. Il était entendu que les documents touchant à la guerre civile (1936-1939) demeuraient exclus du prêt. Le problème porta sur les documents "immédiatement antérieurs au conflit" susceptibles d'être déposés en Catalogne. Mais à quelle date situer les antécédents de la guerre ? Les historiens représentant l'AGGCE optèrent pour prêter les documents antérieurs à 1931, excluant donc tous ceux produits pendant la République (1931-1939). Ils introduisaient ainsi un nouvel élément de confusion : la sélection des documents en fonction de leur valeur "d'antécédence" au conflit. Les historiens catalans fixèrent l'antécédence à octobre 1934, quand la Generalitat fut momentanément suspendue pour avoir refusé d'appliquer des lois républicaines. Un second point de divergence concernait la nature institutionnelle des documents concernés. Pour les historiens catalans, il fallait non seulement réclamer les archives de la Generalitat mais de manière générale, ceux des autres entités et associations existantes en 1936. Pour Antonio Elorza, cette opinion ouvrait la voie à des restitutions en cascade qui finiraient par démanteler le centre salamantin. En janvier 2002, la rupture fut consommée ${ }^{16}$.

\footnotetext{
${ }^{13}$ El País, 03/06/2000, "El Gobierno se niega a trasladar archivos de la guerra civil".

${ }^{14}$ El País, 04/06/2000, Antonio Elorza, "Salamanca/Barcelona : otra vez el archivo".

15 Elle comprenait Carlos Dardé (Université de Cantabrie) et Antonio Morales (Université de Salamanque) pour le Comité, Borja de Riquer (Université autonome de Barcelone) et Joan B. Culla (Idem) pour la Generalitat. Cf. El País, 18/11/2000, "I".

${ }^{16}$ El País, 25/06/2002, "La comisión sobre el fondo catalán del Archivo de Salamanca evidencia hoy su desacuerdo". El País, 24/07/2002, Antonio Elorza, "De nuevo Salamanca".
} 
L'échec des négociations suscita immédiatement une réaction importante en Catalogne : la «Commission de la Dignité » réunit alors des comités citoyens favorables à la restitution $^{17}$. En mai, 500 personnalités appartenant à 205 universités de 47 pays appuyèrent l'initiative : Noam Chomsky, Paul Preston ou Rigoberta Menchu signèrent contre ce que le président de la Generalitat, Jordi Pujol, considéra comme l'illustration du "droit de conquête" ${ }^{18}$.

Vu le désaccord de la commission technique, la nouvelle ministre de la Culture, Pilar del Castillo (PP), déclara en juillet 2002 "[qu']aucun document ne sortira[it] de Salamanque". "La question était close"19. Alléguant d'une très discutable "unité d'archives", elle fondait également sa décision sur une interprétation partisane des recommandations de l'UNESCO ${ }^{20}$. Il s'agit en fait de propositions à débattre présentées par un groupe de travail de l'organisme international sur la conservation des fonds de services secrets, de corps paramilitaires et de tribunaux d'exception. Ces propositions avancent quatre nécessités : l'identification des fonds, l'inventaire des documents à restituer, leur reproduction et la restitution. Ces normes s'inscrivent dans la continuité des travaux que l'UNESCO et le CIA (Conseil international des archives) développèrent à partir des années 1970 afin de régler le sort des archives de guerre par des conventions internationales, sans jamais perdre de vue l'objectif de la réconciliation. Pour Joan Culla, membre de la commission technique désavouée, "quelle différence y a-t-il entre le pillage artistique ordonné par Goering et le pillage documentaire contemporain commandé par Franco ? Est-ce parce que Goering termina sur les bancs de Nüremberg alors que Franco mourut dans son lit?". Plus loin, il ajoutait : "Peut-on condamner Franco et absoudre son œuvre ?" 21 .

Ainsi, le PP n'envisageait le problème que sous l'angle technique ou culturel alors qu'il s'agissait d'un problème politique. Professant un espagnolisme revigoré et un centralisme renforcé, il obligeait la Generalitat à recourir à la voie judiciaire puisqu'elle

\footnotetext{
${ }^{17}$ Commissió de la Dignitat, Volem els papers, La lluita per a la repatriació dels "Papers de Salamanca", Lleida, Pagès ed., 2004. On trouve dans cet opuscule publié par l'association créée en janvier 2002 une chronologie très utile des événements.

${ }^{18}$ El País, 29/01/2005, "100 personalidades respaldan la devolución de los "papeles de Salamanca".

${ }^{19}$ El País, 23/07/2002.

${ }^{20}$ El País, 12/10/2002, "El retorno de los archivos a Cataluña". Pétition de 60 universitaires.
} 
était l'héritière du gouvernement autonome républicain. Quant aux individus encore vivants et aux personnes juridiques renaissantes (syndicats, partis, associations culturelles, etc.), ils se lancèrent dans la même voie au nom du respect du droit de propriété $^{22}$. C'est la mémoire démocratique qui surgissait alors, arguant d'une continuité par-delà le franquisme. Javier Tusell s'intérrogeait avec raison : "la guerre civile est-elle vraiment terminée ?"

Derrière la question des "papiers", c'est toute la démocratie espagnole qui réfléchissait sur ses origines alors que depuis 2000 , montaient de toute part les voix des victimes de la répression. Le $\mathrm{PP}$ avait manqué intentionnellement une occasion de réparer les blessures de la guerre comme il avait toujours refusé de voter au Parlement la condamnation officielle du coup d'État de juillet 1936.

\section{La trêve?}

Jusqu'à présent, la "guerre des papiers" n'avait mobilisé que l'opinion publique catalane et castillane. La fin de non-recevoir du gouvernement alluma un feu qui embrasa toute l'Espagne. En Pays valencien, des entités culturelles entrèrent dans la danse ${ }^{23}$. En Aragon à l'inverse, l'opinion se mobilisa pour défendre l'idée d'un rapatriement à Saragosse des archives de la Couronne d'Aragon, sises à Barcelone. La Generalitat fut accusée de s'approprier un patrimoine culturel et d'entretenir un discours faussement victimiste $^{24}$. En Catalogne aussi, des voix se firent entendre pour tenter de briser le cercle vicieux d'une tension artificiellement alimentée. L'opinion se fatiguait du discours victimiste qu'avait soutenu Jordi Pujol depuis 1980, pendant cinq législatures consécutives. Les élections de l'automne 2003 qui portèrent à la Generalitat le socialiste catalaniste Pascual Maragall changèrent la donne.

Après 2002, les fronts politiques se scindèrent. Au PP, la branche catalane opérait un virage régionaliste inattendu après une longue guerre des chefs. Elle se comptait

${ }^{21}$ El País, 27/07/2002, Joan Culla, "Salamanca y el rey Canuto". Voir aussi El País, 07/05/2004, Joan Culla, "¿Condenar a Franco y absolver su obra?".

${ }^{22}$ Par exemple, Esquerra Republicana de Catalynua, parti indépendentiste, le 1er août 2002, puis Iniciativa per Catalunya-Verds, coalition écolo-communiste, le 9 octobre et Unió, parti catalaniste conservateur, le 10 décembre.

${ }^{23}$ Par exemple, l'Acció cultural del Pais Valencià, le 19/10/2002.

${ }^{24}$ El País, 18/10/2002, "El retorno de los archivos a Cataluña, no". 
désormais au nombre des partisans de la restitution, provoquant la fureur de ses homologues castillans. En Pays valencien aussi, le PP se dit favorable à la récupération des archives de la guerre. Au PSOE, la tendance centraliste perdit du terrain au profit de la minorité fédéraliste que Pascual Maragall symbolise : alors que le PSOE de Salamanque continuait cependant à réclamer l'unité des archives, un jeune chef socialiste, José Luis Zapatero pariait alors sur les tenants d'une décrispation politique. Il emportait l'assentiment des Espagnols, en mars 2004.

Pour la première fois, la concordance politique en Catalogne et à Madrid augurait un règlement rapide de la question des "papiers de Salamanque". Le 18 mai 2004, le Congrès des députés exigea de trouver un accord avant la fin de l'année. À nouveau, la Junte nationale des archives se réunit pour nommer un comité de 18 experts sous la présidence de Federico Mayor Zaragoza, ex-président de l'UNESCO ${ }^{25}$.

Le 24 décembre 2004, le président énonça les conclusions de ce second comité ${ }^{26}$. Il rappelait tout d'abord la nécessité de la conservation, de la description et de l'accessibilité des archives. Le comité reconnaissait le droit de tous les particuliers et institutions à réclamer les documents leur appartenant, à la seule condition qu'ils aient existé en 1936. Les conclusions se fondaient notamment sur le droit international en matière de restitution d'œuvres d'art dans les conflits armés. Ainsi, les "507 cartons de la discorde" qui représentent 2,5\% des 20000 caisses entreposées à Salamanque, devaient retourner en Catalogne ${ }^{27}$. Le comité recommandait enfin la transformation des Archives de Salamanque en centre de recherche international et le rapatriement à Salamanque des papiers dispersés au gré de leur utilisation (archives de la présidence du Gouvernement, archives de la Causa general, archives des conseils de guerre, etc.).

Concrètement, le comité réglait le problème des 507 caisses de documents produits ou reçus par la Generalitat et le Parlement de Catalogne entre 1931 et 1939, émanant principalement des départements de justice (213 caisses), de défense (170), d'économie,

\footnotetext{
${ }^{25}$ Il s'agit de Juan Pablo Fusi, Borja de Riquer, Julio Valdeón, Eugenio García Zraza, Edwards Malefakis, Rosa López Alonso, Pedro González García, Angels Bernal, José Ramón Cruz Mundet, Tomas de la Quadra-Salcedo, Pedro Cruz Villalón, Josep Cruanyes, Federico Mayor Zaragoza, Antonio Morales Moya, Joan Rigol, Julio Valdeón Baruque, Antonio González Quintana.

${ }^{26}$ El País, 24/12/2004, "El comité de expertos aconseja devolver a la Generalitat sus papeles de Salamanca". Et El País, 29/12/2004, "507 archivadores con documentos de la Generalitat entre 1931 y 1939".
} 
de culture, d'agriculture et de santé. S'y trouvent en particulier les actes des sessions parlementaires, le texte du statut d'autonomie de 1932, la correspondance des présidents Macià et Companys, la documentation relative aux activités du comité d'expropriation pendant la guerre, les listes des patrouilles de contrôle, celle des détenus, etc.

Juridiquement, le comité reconnut le droit des victimes et proclama le droit à la réparation. Il souligna en particulier le caractère violent de la spoliation et rappela les normes qui devaient accompagner toute expropriation dans un État de droit. L'unité des Archives fut récusée non seulement à cause de l'extraordinaire hétérogénéité des fonds mais surtout parce qu'elles ne constituèrent jamais qu'un fichier de "l'anti-Espagne", pour reprendre la terminologie franquiste. Enfin, la Generalitat qui réclama ses papiers de manière précoce et continue fut l'unique communauté autonome autorisée à récupérer des archives puisque la seule existante en 1936. Par contre, aucune limite juridique ne permit de limiter les réclamations privées (ce qui ne dépasserait pas, dans tous les cas, $5 \%$ du total des archives salamantines $)^{28}$.

Ces conclusions "équilibrées" n'effacent cependant pas toutes les inquiétudes. Premièrement, d'autres communautés que la catalane récusent l'argument de la continuité institutionnelle des deux Generalitat : en effet, peut-on soutenir que la légitimité du gouvernement autonome catalan repose sur des lois républicaines et non pas sur la constitution de 1978 ? La communauté valencienne et surtout l'Euskadi entreprennent d'ores et déjà de contester le comité, arguant du fait qu'un gouvernement basque exista sous la Seconde République ${ }^{29}$. Toutefois, selon Tomas de la QuadraSalcedo, professeur de droit administratif à l'université Carlos III, il n'y aurait pas de continuité historique entre les deux gouvernements basques...

Deuxièmement, le comité laisse la porte ouverte aux réclamations privées. Le problème n'affecte pas les partis ni les syndicats car une loi de 1978 l'avait réglé en son temps, en offrant un délai de six ans pour réclamer les restitutions (c'est-à-dire jusqu'en décembre 1984). Par contre, les municipalités et les personnes privées peuvent recourir. Certains historiens ont critiqué ces mesures qui laissent la voie libre à un démantèlement

\footnotetext{
${ }^{27}$ El País, 09/01/2005, Andrés Padilla, "Las 507 cajas de la discordia".

${ }^{28}$ El País, 31/12/2004, José Alvarez Junco, "Expertos y papeles".

${ }^{29}$ On trouve à Salamanque la correspondance privée du premier Lehendakari, José Antonio Aguirre, et celles des chefs historiques du parti nationaliste basque (PNV), Juan de Ajuriaguerra et Manuel Irujo.
} 
important du fond salamantin. Mais aucune disposition juridique ne peut empêcher les propriétaires légitimes à recouvrer leurs biens...

Troisièmement, trois membres castillans du comité ont défendu la pertinence de l'unité d'archive $^{30}$. Pour la majorité du comité, comme le dit Juan Pablo Fusi, "la seule unité est que ces documents se trouvent dans le même bâtiment". Pour les contestataires, l'intégrité des archives doit être sauvegardée" afin qu'elles servent de témoin de la répression". En effet, comme le rappelle Antonio Elorza, la qualité de "butin" concerne autant les fonds catalans que ceux pris à Madrid, à Bilbao ou à Valence. Pour lui, la prétention de la Generalitat repose sur une analyse erronée selon laquelle en Espagne, ce sont les gauches qui ont perdu la guerre tandis qu'en Catalogne, c'est tout le monde. Or, on sait bien qu'il y eut des intérêts économiques, politiques et culturels catalans pour s'enthousiasmer de l'entrée des troupes franquistes à Barcelone...

Les conclusions du comité qui servit de base à la résolution gouvernementale en faveur de la restitution ne pouvaient éviter le renouveau de "la guerre des papiers". Dès le 23 décembre, les Cortes de Castille et Léon changèrent la loi locale des archives et celle du patrimoine afin de "blinder" les Archives de Salamanque, selon leur propre expression $^{31}$. Mais cette communauté n'a pas compétence législative sur des archives de l'État, ce qui entraîna automatiquement un recours du Gouvernement central devant le Tribunal constitutionnel. La loi du patrimoine tenta d'élever les "papiers de Salamanque" au rang de Bien d'Intérêt Culturel pour en limiter la possibilité de déplacement $^{32}$. La Junte de Castille choisit donc d'entrer dans une bataille politique et juridique contre "un attentat culturel", "un pillage du patrimoine castillan" qui pourrait conduire au "démantèlement de l'Espagne". Jouant des mémoires de la guerre civile, un membre influent du PP déclarait que "le PSOE se lie à nouveau avec les séparatistes et les communistes pour rompre l'unité des Archives de la guerre civile". Il dénonçait la faiblesse du Gouvernement central devant les exigences catalanes et "le courant de

\footnotetext{
${ }^{30}$ Il s'agit de Julio Valdeón, historien désigné par la Junta de Castilla y León, de Eugenio García Zarza, représentant la municipalité de Salamanque et de Antonio Morale Moya, professeur à l'université de Salamanque et ancien membre de la commission technique de 2000. Cf. El País, 09/01/2005, "El polémico informe".

${ }^{31}$ El País, 23/12/2004, "Las Cortes de Castilla y León "blindan" el Archivo de Salamanca". El País, 24/12/2004, "Satisfacción general en Cataluña mientras en Salamanca se prepara la resistencia". El País, 31/12/2004, "El Gobierno de Castilla y León inicia una batalla "jurídica y política" por el Archivo".

${ }^{32}$ En 1988, le Tribunal constitutionnel donna raison à la Junte de Andalucía contre l'État afin de bloquer le déplacement des archives Medinacelli.
} 
révision de l'histoire qui menace de faire peser les soupçons sur le patrimoine culturel de tous les Espagnols". La très réactionnaire Académie royale d'histoire prit le parti de défendre les agissements de la Junte de Castille, dans la parfaite continuité de la révision du contenu des programmes scolaires dans un sens résolument centraliste et autoritaire qu'elle avait entrepris sous le règne des conservateurs ${ }^{33}$. Le maire de Salamanque déclarait en nouveau Cid Campeador que "d'ici, il ne sortira aucun papier". La rhétorique nationaliste espagnole de tradition anticatalaniste en appelait donc à la patrie en danger. Dans El País du 26 décembre 2004, Javier Tusell douta : “ ¿Conflicto concluido ?" 34 .

Les papiers de la paix? Enjeux.

La complexité de l'affaire des "papiers de Salamanque" soulève bien évidemment plusieurs problèmes et ravive de vieilles lignes de fracture. Il faut d'abord souligner que l'affaire ne comprend d'enjeux ni historiographiques ni archivistiques.

D'un point de vue historiographique, les historiens ont été relativement solidaires même si des divergences de vue se sont manifestées, notamment en 2002. Le comité de 2004, présidée par Federico Mayor Zaragoza a mis en valeur la fonction d'expertise des historiens en même temps que leur cohésion (le rapport n'a pas eu de vote contre et seulement trois abstentions). L'opposition n'a pas manqué de dénoncer le parti-pris de la commission du comité, en vain. Ces professionnels (historiens et archivistes) ont bénéficié du vent de critique qui a touché la commission d'enquête des attentats terroristes du 11 mars 2004, uniquement composée d'hommes politiques. De plus, les historiens ont rappelé à plusieurs reprises qu'à leurs yeux, la localisation des archives avait peu d'importance au regard des problèmes de conservation et d'accès aux documents. Pour eux, la technologie moderne de reproduction supplée les inconvénients de la dispersion documentaire, même s'ils souhaitent que les reproductions soient concentrées dans un unique lieu, à Salamanque. Les historiens spécialistes de la guerre civile ont également écrit que les Archives de Salamanque étaient une source

\footnotetext{
${ }^{33}$ El País, 25/01/2005, "La Academia de Historia pide que el Archivo de Salamanca siga unido".

${ }^{34}$ El País, 26/12/2004, Javier Tusell, "¿Conflicto concluido?".
} 
d'information bien inférieure aux archives militaires par exemple. Pour Santos Juliá par exemple, "ce conflit est totalement anachronique" 35 .

Aux yeux des archivistes, les premières dissensions se sont tues au profit d'un consensus professionnel. Le souci d'éviter un démantèlement trop important tout en réparant les droits des victimes guida donc leur travail. Un document de juin 2004 rédigé par la Confédération des associations d'archivistes, bibliothécaires, muséologues et documentalistes affirmait la nécessité de reconnaître le droit légitime des victimes et de respecter les fonds actuels qui représentent "un témoignage exceptionnel de ce que fut la répression en Espagne de 1937 à 1963"36 . Les Archives de Salamanque sont qualifiées de "lieu de mémoire" espagnol dont la dispersion serait "une perte irréparable". Comment assumer la réparation sans démembrer physiquement les fonds ? Ce texte proposait de reconnaître de manière intangible la propriété des fonds spoliés mais de limiter d'éventuelles restitutions à la condition que les documents soient reproduits et accessibles dans des archives publiques (sous forme de dépôts). Cette tentative de conciliation regrettait l'absence de norme générale concernant les réparations aux victimes du franquisme, les lois n'agissant qu'au cas par cas (la loi sur la restitution des biens aux syndicats en 1986, par exemple). Pour l'Association des archivistes de Castille et Léon, le débat professionnel a été malheureusement supplanté par des considérations politiques hors de $\operatorname{propos}^{37}$. Le maire de Salamanque, si prompt à défendre les Archives de la guerre civile, laisse pourrir les fonds municipaux dans des caves insalubres. Les normes archivistiques de l'État et de la Castille n'ont jamais été unifiées. Comment l'institution autonome qui n'a jamais appliqué ses propres lois archivistiques datant de 1991 pourrait-elle prétendre gérer les fonds appartenant à d'autres ? Les archives constituent un "élément indispensable de la mémoire des entités qui l'ont produite et des sociétés dont elles émanent et un instrument fondamental pour construire l'histoire". Dans un État de droit, les archives sont de plus des preuves indispensables pour le maintien des droits et des devoirs des citoyens et des administrations : "Négliger les archives, c'est faire obstacle à l'exercice des droits démocratiques de tous les citoyens".

\footnotetext{
${ }^{35}$ El País, 04/06/2004, "El presente : un inmenso barullo".

${ }^{36}$ CAABMD, "Bases para un debate profesional en torno al Archivo General de la Guerra Civil", Hoja informativa, mayo-junio $2004, \mathrm{n}^{\circ} 186$.

${ }^{37}$ Comunicado de la asociación de archiveros de Castilla y León (ACAL) sobre la polémica del Archivo general de la guerra civil española, décembre 2004.
} 
On voit donc que l'affaire a renforcé l'autonomisation et la professionnalisation des historiens et archivistes. Les enjeux principaux se situent donc sur un autre plan : politique, symbolique et mémoriel.

Les usages des "papiers de Salamanque" au gré des aléas politiques n'ont pas manqué de part et d'autre. On a vu les socialistes changer d'opinion en 1996 alors que les catalanistes s'emparaient du problème pour conjurer un déclin électoral inéluctable. Aujourd'hui, le Parti populaire, déstabilisé par les mensonges de José María Aznar en mars 2004, cherche un cheval de bataille pour se requinquer. Son fief vieux-castillan (Salamanque-Valladolid) et madrilène lui est entièrement acquis. Par ailleurs, depuis deux ans, le Gouvernement doit faire face à la fronde institutionnelle de l'Euskadi qui cherche, par l'intermédiaire du Lehendakari (président) Ibarretxe (on peut supprimer ce qui est souligné, pour alléger) à promouvoir un statut d'association entre l'État espagnol et un éventuel État basque. La modération des Catalans qui ne remettent pas en cause l'unité proclamée dans l'article premier de la Constitution est un atout considérable dans la main de José Luis Zapatero pour écarter le danger d'une sécession basque. Les événements basques déterminent largement le climat de tension entre le Gouvernement et l'opposition, traditionnellement réactive à ce problème et plus encore depuis que les attentats du 11 mars, faussement attribués à ETA, lui ont coûté le pouvoir.

Mais la "guerre des papiers" a ravivé plus profondément une ancienne fracture politique divisant deux lectures antagonistes de l'Espagne : d'une part, une lecture unitaire défendue par les conservateurs et une majorité des socialistes ; d'autre part une lecture fédéraliste défendue par les partis nationalistes, le parti communiste et une partie du parti socialiste. José Luis Zapatero a clairement pris parti pour l'Espagne plurielle que le Parti socialiste catalan désormais au pouvoir appelait de ses vœux. Sa lecture de la Constitution de 1978 encourage l'Espagne des autonomies, la révision des statuts d'autonomie octroyés au début des années 1980 à la Catalogne et au Pays basque et la conversion du Sénat en chambre des régions sur l'exemple états-unien. Cette option représente un virage pour le PSOE qui défend traditionnellement des positions centralistes, en particulier dans ses fiefs d'Estrémadure et de Nouvelle Castille. Le tournant, en partie imputable à l'évolution des socialistes andalous désormais favorables au renforcement de la Junte d'Andalousie, s'explique également comme réponse au ton 
espagnoliste que le PP a adopté depuis 2000. De nombreuses manifestations du centralisme de l'État ont contribué à alimenter la querelle centenaire.

Sous-jacent au thème de l'unité nationale/archivistique se manifeste un autre problème politique : la remise en cause de la transition démocratique, entre 1975 et 1982. L'État des autonomies résulte d'un fragile équilibre qui consista à noyer les revendications particularistes basque et catalane dans un cadre général qui promouvait 17 régions autonomes ; ce que les Espagnols ont résumé par la formule non dénuée d'humour de "café para todos" (du café pour tout le monde). En fait, ce principe a rapidement entraîné une course revendicatrice au nom d'un alignement sur les communautés les plus dotées, les trois "communautés historiques" basque, catalane et galicienne. L'inquiétude d'un démantèlement des Archives plonge donc ses racines dans un effet pervers du pacte constitutionnel. C'est pourquoi le comité d'experts n'a reconnu légitimes que les revendications catalanes, au nom d'une continuité institutionnelle problématique. La restriction n'a pas l'air d'arrêter les Valenciens ou les Aragonais.

Plus généralement, ce sont les conditions politiques d'une transition démocratique qui sont ici discutées. Les Espagnols découvrent les ombres d'un processus de démocratisation qu'ils considéraient jusqu'à présent comme parfait, au point de le présenter comme modèle de transition dans les pays d'Europe orientale. Comme le déclara le président de la Fondation Pablo Iglesias qui conserve les archives du PSOE, Aurelio Martín Nájera : "Peut-être que si en 1977, on avait reconnu que les Archives [de Salamanque] étaient le fruit d'une spoliation et qu'on avait intégré dans le Comité directeur de l'AGCCE [formé en 1999] des représentants des victimes, on ne serait pas arrivé à la situation de crispation actuelle". "Le problème, ajoute José Andrés Rojo, est que la transition modèle n'a pas appelé les choses par leur nom et des archives constituées à partir de la rapine franquiste se sont converties en lieu de mémoire du conflit civil" ${ }^{\prime 38}$. Il ne faut pas voir dans cette conversion un fait du hasard : la transition espagnole s'est construite sur le déni du legs républicain, de la mémoire de la guerre civile et plus encore de celle de la répression. Comme le remarque la Confédération des associations d'archivistes, "à la différence d'autres transitions politiques, on n'envisagea jamais dans la nôtre l'existence d'un quelconque organisme qui assume les réparations

\footnotetext{
${ }^{38}$ El País, 04/06/2004, José Andrés Rojo, "La memoria de la represión". Les propos de Martín Nájera sont également tirés de cet article.
} 
aux violations des droits commis par le régime répressif. Il ne se constitua jamais aucune commission de la Vérité" ${ }^{39}$. Les années 2000 marquent donc dans l'opinion publique espagnole une critique de la transition et une dénonciation acerbe du silence entretenu par une génération politique qui souhaitait avant toute chose, réconcilier les Espagnols autour d'un projet politique d'avenir.

Dans ces conditions, on comprend que l'enjeu politique soit d'essence symbolique et mémorielle, ce que tous les protagonistes ont aisément reconnu. Plus encore que la mémoire de la guerre civile, c'est la mémoire de la répression qui est en cause. De la part des victimes, un immense besoin de justice s'est fait ressentir dans toutes les couches de la société, avec une vigueur qui a surpris les observateurs. Depuis 1999, une marée du souvenir et de parole refoulée a littéralement submergé l'Espagne sous des formes diverses : travaux historiographiques novateurs, séries télévisées et radiophoniques, innombrables romans dont font partie les fameux Soldats de Salamine de Javier Cercas, vendus à 450000 exemplaires; monuments commémoratifs et destructions de l'appareil symbolique franquiste parfois encore en place, création de nombreuses associations d'anciens prisonniers ou bien d'enfants de victimes, d'associations culturelles de tout type travaillant à la "récupération de la mémoire historique", notamment aux fouilles des fosses communes dans près de 10000 villages répartis dans tout le pays. Mais à la différence de la vague de mémoire qui submergea la France entre 1971 et 1996 à propos de la collaboration d'État au génocide juif, aucune poursuite judiciaire n'est possible, à cause de la loi d'amnistie générale votée en 1976... sur pression des démocrates qui réclamaient alors la libération des prisonniers politiques. Les Espagnols découvrirent pour la plupart l'extrême violence d'une répression qui avait touché un demi-million de personnes, un système implacable de 198 camps de concentration qui pratiqua à une large échelle le travail forcé. Quelque 10 000 enfants de républicains furent séparés de leurs parents, débaptisés puis réattribués à de nouveaux foyers, notamment des couvents. En 2002, les associations portèrent devant l'ONU la résistance de l'État espagnol à l'ouverture des fosses communes. C'est seulement le 20 novembre 2002, 27 ans après la mort du dictateur, que le Congrès condamna le coup d'État du 18 juillet 1936.

\footnotetext{
${ }^{39}$ CAABMD, op. cit.
} 
La guerre des "papiers de Salamanque", on le voit, ne prend toute sa dimension que dans un contexte bien particulier. Le besoin de justice se double d'un effort pédagogique considérable qui vise à une reconnaissance globale des droits des victimes. Comment justifier dans une démocratie l'offense que représente le maintien des Archives de Salamanque La restitution y est qualifiée de "geste de paix, de pédagogie et de générosité politique" et de "réparation des victimes"40, nécessaire pour "fermer les vieilles blessures"41. À travers les archives, la démocratie espagnole découvre ses origines, celle d'une république démocratique et sociale qu'on tendait à oublier parce qu'on attribuait le déclenchement de la guerre à son échec, non sans quelque raison.

Pour la première fois, les "papiers" de Salamanque pourraient être ceux de la paix. En Catalogne, où le parti nationaliste conservateur épuisa toutes les ressources de la victimisation collective pour justifier sa politique, l'heure est à l'apaisement des conflits nationalistes. Pour la première fois, certaines voix isolées osent proposer d'assumer la guerre civile et la répression comme un patrimoine douloureux mais commun. "Pourrons-nous, une fois pour toutes, réparer tant de mépris, tant d'humiliation sans que personne ne se sente agressé?" interroge Joaquim Puig ${ }^{42}$. Au-delà du problème de la restitution de biens spoliés, c'est bien la capacité des Espagnols à assumer ce passé commun qui est en jeu. Eduardo Haro Tecglen conclu : "La guerre civile n'est à personne, ou bien elle est à tout le monde. [...] [Ces archives] ne sont pas de Salamanque ni de Barcelone mais de tout cet ensemble historiquement malchanceux que l'on peut appeler l'Espagne"43. Et si les archives n'étaient pas un butin de guerre? Pour Antonio Elorza, "il ne viendrait à l'idée de personne de retirer d'un musée de l'holocauste les objets des Séfarades ou des juifs hongrois parce que ces derniers se sentent particulièrement affectés" ${ }^{44}$. Les nationalismes espagnol et catalan ont brouillé les pistes en alimentant un combat sans fin alors que les Archives de Salamanque

\footnotetext{
${ }^{40}$ El País, 16/07/2004, Dídac Martínez, "Una reflexión política y una solucioón tecnológica".

${ }^{41}$ El País, 04/06/2004, José Andrés Rojo, "La memoria de la represión".

${ }^{42}$ El País, 27/12/2004, Joaquim Puig, "Papeles para la paz" ; 01/06/2004, Rosa Montero, "Mudanzas".

${ }^{43}$ El País, 31/12/2004, Eduardo Haro Tecglen, "La guerra de los papeles".

${ }^{44}$ El País, 30/12/2004, Antonio Elorza, "El archivo como botín".
} 
pourraient être, selon Javier Tusell, "le point de départ d'un exercice de réconciliation" 45 .

Mais ce qu'il manque peut-être au débat espagnol, c'est un recul critique forcément salutaire. On pourrait s'étonner de constater qu'aucune des parties n'a remis en cause l'opportunité d'un centre d'archives de la guerre civile, comme s'il allait de soi que cet événement méritait un traitement qui l'isolerait du reste de l'histoire espagnole. On peut également douter de l'efficacité d'une mémoire qui se veut pédagogique et moralisatrice. Enfin, si l'accès et la reproduction sont assurés, à quoi bon posséder quelques cartons de papiers originaux ? On peut légitimement se demander à partir de quand les documents demandés ont acquis cette valeur fétichiste dont les imaginaires collectifs se sont avidement emparés. De fait, ce qui fonde la valeur des 507 cartons n'est pas tant leur spoliation que leur usage postérieur pour la répression. Ce ne sont pas les papiers qui comptent mais l'usage qu'on en fit. On peut sérieusement douter que leur restitution rétablisse en quoi que ce soit cet usage infamant. L'historien Enric Ucelay-Da Cal pense ainsi que l'adoration des biens culturels finit par attribuer une valeur surnaturelle à des documents devenus des biens spirituels et patrimoniaux ${ }^{46}$. L'idolâtrie des archives ont fait des "papiers de Salamanque" les nouvelles reliques de l'Espagne démocratique.

\footnotetext{
${ }^{45}$ El País, 26/12/2004, Javier Tusell, "¿Conflicto concluido?".

${ }^{46}$ El País, 16/07/2004, Enric Ucelay-Da Cal, "Un relicario me voy a hacer".
} 